SU-ITP-42

October 8, 2018

\title{
Reissner-Nordstrom Black Holes and Strings with Rescaled Tension
}

\author{
EDI HALYO * \\ Department of Physics \\ Stanford University \\ Stanford, CA 94305
}

\begin{abstract}
We show that extreme and nonextreme Reissner-Nordstrom black holes in five dimensions can be described by closed fundamental strings with two charges in a magnetic five brane. Due to the five brane background the string oscillator number and tension are rescaled whereas the mass remains fixed. The black hole mass is given by the sum of the five brane and string masses. Its entropy however is given by only that of the string with the tension rescaling taken into account. We also show that the emission of a low energy scalar from the slightly nonextreme string matches the Hawking radiation expected from the black hole.
\end{abstract}

* e-mail address: halyo@dormouse.stanford.edu 


\section{Introduction}

During the last year there has been remarkable progress in understanding the microscopic structure of Ramond-Ramond (RR) charged black holes in type II string theory by using the D-brane technology[1]. These black holes are made of a collection of different types of D-branes with the mass of the black hole[2,4]. By counting the open strings which end on different D-branes one can count the number of states of the system which reproduces the black hole entropy. This was done for five and four dimensional extreme and nonextreme black holes $[2,3,5]$ (and also for rotating ones[6]). Furthermore, emission of low energy scalars from the D-brane configuration was shown to match the Hawking radiation expected from the corresponding black hole[7]. The picture that emerges from these works is as follows. Extreme black holes are very well described by noninteracting BPS states of a long (D) string[8]. For small deviations from extremality, the essential degrees of freedom of the black hole are weakly interacting non BPS excitations of a long closed (D) string. This is also true for low energy processes such as radiation of scalars from the black hole. Other branes present such as five branes do not play a dynamical role but simply restrict the string oscillations to lie inside their world-volume. Thus, the near extreme black hole seems to behave like a weakly interacting system in one dimension which has a close resemblance to a (D) string.

On the other hand, it was appreciated for some time that a sufficiently massive string state becomes a black hole since its Schwarzschild radius becomes smaller than its size [9]. Even though state counting for strings is well-known, a simple connection between black holes and strings has not been established. This is mainly due to the fact that naively the entropy of a string is proportional to its mass whereas for the black hole $S_{B H} \sim M^{2}$. This apparent discrepancy was eliminated in ref. [10]. There it was shown (for Schwarzschild black holes) that a string in its own gravitational field (or Rindler space) has a Rindler energy which gives the correct scaling for the black hole entropy. This result was later expanded for Schwarzschild black holes in all dimensions with the correct coefficient[11]. Another potential problem for the stringy description of black holes is the fact that a string 
can carry at most two charges whereas a black hole can have more. This requires the introduction of another object into the description as we will see below.

In this paper, we consider Neveu-Schwarz (NS) charged Reissner-Nordstrom (RN) black holes in five dimensions (in type II string theory compactified on $T^{5}$ ). We show that these black holes can be described by closed fundamental strings with two charges in a magnetic NS five brane background. The essential degrees of freedom of the black hole are the string states. The extreme and nonextreme black holes are described by the BPS and non BPS states of the string respectively. The five brane background restricts the string oscillations to lie in its world-volume and provides the Rindler space-time due to which the string oscillator number and tension are rescaled. The mass of the black hole is given by the sum of the masses of the five brane and the string. Naively the string does not reproduce the black hole entropy. This is not surprising since the naive string entropy formula holds for a free string whereas we have a string in a strong gravitational background. In order to find the entropy, we calculate the Rindler energy of the string in the five brane background and identify $S=2 \pi E_{R}$. This method gives the correct black hole entropy for $c_{e f f}=6$. We interpret this effective central charge to mean that the string oscillations are confined into the world-volume of the five brane. Since $E_{R}$ is dimensionless and has the above relation to entropy we identify it with the (square root of the) string oscillator number in Rindler space. Comparing this with the free string we find that the oscillator number is rescaled due to the gravitational background. This scaling factor is exactly what is needed to transform the naive string entropy into that of the black hole. On the other hand, the string mass (added to the five brane mass) reproduces the correct black hole mass. We can hold the string mass fixed under a rescaling of the oscillator number only if we rescale $\alpha^{\prime}$ (or the string tension, $T=1 / 2 \pi \alpha^{\prime}$ ) by the same factor. ${ }^{\star}$ Thus, we conclude that the effect of the five brane background on the string is to rescale its oscillator number and tension by the same factor while the string mass remains

$\star$ For strings with rescaled tension in a somewhat different context see [12]. 
fixed. This picture can be considered $\mathrm{S}$ dual to that of ref. [SV] in terms of D branes in type IIB string theory.

Our main assumption is that all gravitational effects in the black hole are summed up in the rescaling of the oscillator number and the string tension due to the five brane background. The string mass remains fixed, i.e. in the black hole it is equal to its value for the free string both for BPS and non BPS states. This makes sense for the BPS states but not for the non BPS ones which is reminiscent of the nonextreme cases in the D-brane picture. We have no explanation for this other than pointing out that the method works for both extreme and nonextreme RN black holes in five dimensions. Here, we are also neglecting any possible effects due to the mass of the string on itself. This is justified for a black hole with one charge (a five brane) since the string is a small perturbation on this background. On the other hand, for a RN black hole, the string has two charges and weighs twice as much as the five brane. In this case, it is hard to understand why these effects are absent. Perhaps a deeper understanding of these issues may be reached by investigating strings in Rindler space-times [13] and/or by considering more fundamental descriptions of the near horizon region along the lines of refs. [14].

The paper is organized as follows. In section 2, we review the classical RN black hole solution in type II string theory. We define the parameters of the string picture in terms of the parameters of the classical solution. In section 3, we first review the case of a five dimensional black hole with one charge, i.e. a five brane. We introduce our method in detail for this simpler case and show that small deviations from extremality are described by a neutral string with a rescaled tension. We then consider extreme and nonextreme $\mathrm{RN}$ black holes in five dimensions. We show that by using our method their masses and entropies are given by a string with two charges and a rescaled tension in a five brane background. In section 4, we calculate the rate of emission of a low energy scalar from a slightly nonextreme string and show that it matches the expected Hawking radiation exactly. Section 5 contains a discussion of our results and our conclusions. 


\section{The Classical Reissner-Nordstrom Black Hole in Five Dimensions}

In this section, we review the solution for the NS charged RN black hole in five dimensions[3]. The classical solution to the low energy equations of motion in type II string theory compactified on $T^{5}$ is given by the metric $g_{\mu \nu}$, the NS antisymmetric tensor $B_{\mu \nu}$ and the dilaton $g^{2}=e^{-2 \phi}$. The RR three form, the self-dual five form and the RR scalar are set to zero. Also, the asymptotic value of the dilaton $\phi$ is taken to be zero. The classical five dimensional RN black hole metric is given by

$$
d s^{2}=-f^{-2 / 3}\left(1-\frac{r_{0}^{2}}{r^{2}}\right) d t^{2}+f^{1 / 3}\left[\left(1-\frac{r_{0}^{2}}{r^{2}}\right)^{-1} d r^{2}+r^{2} d \Omega_{3}^{2}\right]
$$

where

$$
f=\left(1+\frac{r_{0}^{2} \sinh ^{2} \alpha}{r^{2}}\right)\left(1+\frac{r_{0}^{2} \sinh ^{2} \beta}{r^{2}}\right)\left(1+\frac{r_{0}^{2} \sinh ^{2} \gamma}{r^{2}}\right)
$$

The solution is parametrized by six parameters, $\alpha, \beta, \gamma, r_{0}$ and the compactified one and four volumes $2 \pi R$ and $(2 \pi)^{4} V$. The total energy of the black hole is

$$
E=\frac{R V r_{0}^{2}}{2 g^{2} \alpha^{\prime 4}}(\cosh 2 \alpha+\cosh 2 \beta+\cosh 2 \gamma)
$$

The entropy of the black hole is found from the area of the horizon using the Bekenstein-Hawking formula

$$
S=\frac{A_{H}}{4 G_{5}}=\frac{2 \pi R V r_{0}^{3}}{g^{2} \alpha^{\prime 4}} \cosh \alpha \cosh \beta \cosh \gamma
$$

where the ten and five dimensional Newton constants are given by $G_{10}=8 \pi^{6} g^{2} \alpha^{\prime 4}$ and $G_{5}=G_{10} /(2 \pi)^{5} R V$. From the first law of thermodynamics and using eqs. (2.3) and (2.4) we find the Hawking temperature

$$
T_{H}=\frac{1}{2 \pi r_{0} \cosh \alpha \cosh \beta \cosh \gamma}
$$

The RN black hole carries three NS charges

$$
Q_{5}=\frac{r_{0}^{2}}{2 \alpha^{\prime}} \sinh (2 \alpha)
$$




$$
\begin{aligned}
Q_{1} & =\frac{V r_{0}^{2}}{2 g^{2} \alpha^{\prime 3}} \sinh (2 \beta) \\
n & =\frac{R^{2} V r_{0}^{2}}{2 g^{2} \alpha^{\prime 4}} \sinh (2 \gamma)
\end{aligned}
$$

These are the charges of the black hole under the NS three form $H_{3}$, its dual $H_{7}$ and Kaluza-Klein two form coming from the metric. Note that due to the fact that we have a RN black hole the scalars in the solution, i.e. the dilaton $\phi$ and the compactification volumes $R, V$ (which are a priori fields) are constant in space. The $\mathrm{RN}$ condition is $\alpha=\beta=\gamma$ so that the three contributions to the mass of the black hole are equal. The extreme limit is obtained by $r_{0} \rightarrow 0$ and $\alpha, \beta, \gamma \rightarrow \infty$ with the charges $Q_{1}, Q_{5}, n$ fixed. The cases with less than three charges are obtained by setting the corresponding angles to zero.

The properties of the black hole can be written in a suggestive way if we trade the six parameters $\alpha, \beta, \gamma, r_{0}, R, V$ for $N_{1}, \bar{N}_{1}, N_{5}, \bar{N}_{5}, n_{L}, n_{R}$ defined by

$$
\begin{aligned}
& N_{5}=\frac{r_{0}^{2}}{4 \alpha^{\prime}} e^{2 \alpha} \\
& \bar{N}_{5}=\frac{r_{0}^{2}}{4 \alpha^{\prime}} e^{-2 \alpha} \\
& N_{1}=\frac{V r_{0}^{2}}{4 g^{2} \alpha^{\prime 3}} e^{2 \beta} \\
& \bar{N}_{1}=\frac{V r_{0}^{2}}{4 g^{2} \alpha^{\prime 3}} e^{-2 \beta} \\
& n_{L}=\frac{R^{2} V r_{0}^{2}}{4 g^{2} \alpha^{\prime 4}} e^{2 \gamma} \\
& n_{R}=\frac{R^{2} V r_{0}^{2}}{4 g^{2} \alpha^{\prime 4}} e^{-2 \gamma}
\end{aligned}
$$

In terms of the above numbers, the charges of the black hole are $Q_{1}=N_{1}-\bar{N}_{1}$, $Q_{5}=N_{5}-\bar{N}_{5}, n=n_{L}-n_{R}$. The black hole mass is

$$
M_{B H}=\frac{R V}{g^{2} \alpha^{\prime 3}}\left(N_{5}+\bar{N}_{5}\right)+\frac{R}{\alpha^{\prime}}\left(N_{1}+\bar{N}_{1}\right)+\frac{1}{R}\left(n_{L}+n_{R}\right)
$$


The entropy can be written as

$$
S=2 \pi\left(\sqrt{N_{1}}+\sqrt{\bar{N}_{1}}\right)\left(\sqrt{N_{5}}+\sqrt{\bar{N}_{5}}\right)\left(\sqrt{n_{L}}+\sqrt{n_{R}}\right)
$$

The extreme limit is given by $\bar{N}_{1}=\bar{N}_{5}=n_{R}=0$. Small deviations beyond extremality are given by $\bar{N}_{1} \sim \bar{N}_{5} \sim n_{R}<<N_{1} \sim N_{5} \sim n_{L}$. Note that all three anticharges must become nonzero in order to satisfy the RN nature of the nonextreme black hole. The RN condition, $\alpha=\beta=\gamma$ gives two relations which fix two of the moduli

$$
V=\left(\frac{g^{2} N_{1} \bar{N}_{1}}{N_{5} \bar{N}_{5}}\right)^{1 / 2}
$$

and

$$
R=\left(\frac{n_{L} n_{R}}{N_{1} \bar{N}_{1}}\right)^{1 / 4}
$$

Our aim in the next section will be to show that the RN black hole mass and entropy are given by that of an fundamental closed string with a renormalized tension in a five brane background. We will identify $Q_{1}$ and $n$ with the net winding and momentum number of the string whereas $Q_{5}$ will be the net five brane (winding) number. The mass of the black hole will be given by the sum of the background (five brane) and string masses. The entropy, on the other hand, will be given simply by that of the string by properly taking the rescaling of the string tension due to the gravitational effects into account. We will show that this method applies to both the extreme and nonextreme black holes with any number of charges.

\section{Black Holes and Strings with Rescaled Tension}

We begin by examining a five dimensional black hole with only one charge which was done in refs. $[15,11]$. We introduce our method of string tension rescaling for this simple case. We then generalize this to extreme and nonextreme RN black holes with three charges. 


\subsection{Black Holes with One Charge}

Consider a five dimensional nonextreme black hole with one NS charge $Q_{5}=$ $N_{5}$. The metric for this solution is obtained from eq. (2.1) by taking $\beta=\gamma=0$ and is given by

$$
d s^{2}=-f^{-2 / 3}\left(1-\frac{r_{0}^{2}}{r^{2}}\right) d t^{2}+f^{1 / 3}\left[\left(1-\frac{r_{0}^{2}}{r^{2}}\right)^{-1} d r^{2}+r^{2} d \Omega_{3}^{2}\right]
$$

where

$$
f=\left(1+\frac{r_{0}^{2} \sinh ^{2} \alpha}{r^{2}}\right)
$$

In terms of the numbers in eq. (2.7) the mass of this black hole is (here $N_{1}=\bar{N}_{1} \sim$ $\left.n_{L}=n_{R}<<N_{5}\right)$

$$
M_{B H}=\frac{R V}{g^{2} \alpha^{\prime 3}} N_{5}+\frac{R}{\alpha^{\prime}}\left(N_{1}+\bar{N}_{1}\right)+\frac{1}{R}\left(n_{L}+n_{R}\right)
$$

and the entropy is

$$
S=2 \pi \sqrt{N_{5}}\left(\sqrt{N_{1}}+\sqrt{\bar{N}_{1}}\right)\left(\sqrt{n_{L}}+\sqrt{n_{R}}\right)
$$

We want to show that the nonextreme excitations of this black hole are given by a closed long elemenatry string with no charge $[15,11]$ so that

$$
M_{s t}^{2}=\frac{4 N_{L}}{\alpha^{\prime}}=\frac{4 N_{R}}{\alpha^{\prime}}
$$

with the oscillator numbers

$$
N_{L, R}=\frac{1}{4}\left[\left(\sqrt{N_{1}}+\sqrt{\overline{N_{1}}}\right)\left(\sqrt{n_{L}}+\sqrt{n_{R}}\right) \pm\left(\sqrt{N_{1}}-\sqrt{\bar{N}_{1}}\right)\left(\sqrt{n_{L}}-\sqrt{n_{R}}\right)\right]^{2}
$$

Note that $N_{L, R}$ above satisfy the level matching condition for any $N_{1}, \bar{N}_{1}, n_{L}, n_{R}$. The deviation of the black hole mass from its extreme value is given by the string 
mass above. The extreme mass is given simply by that of the five brane background. The entropy is also given solely by that of the string since the entropy of the extreme black hole vanishes. We remind that for the small neutral deviations from extremality $N_{1}=\bar{N}_{1}, n_{L}=n_{R}$ and $N_{1}$. Thus for this case $N_{L}=N_{R}=4 N_{1} n_{L}$ as expected from the level matching conditions for a closed string. The total mass of the black hole is

$$
M_{B H}=\frac{R V}{g^{2} \alpha^{\prime 3}} N_{5}+\frac{2}{\sqrt{\alpha^{\prime}}} \sqrt{\frac{4 N_{1} n_{L}}{\alpha^{\prime}}}
$$

This is the same as eq. (3.3) for all deviations from extremality (in mass) equal, i.e. $N_{1} R / \alpha^{\prime}=n_{L} / R$. Thus, we are able to reproduce the nonextreme black hole mass formula. The black hole entropy is more difficult to obtain however. Naively, the entropy of the black hole is given by that of the string

$$
S=2 \pi \sqrt{\frac{c}{6}}\left(\sqrt{N_{L}}+\sqrt{N_{R}}\right)
$$

Using the expressions for $N_{L, R}$, we see that this does not give the black hole entropy in eq. (3.4). This is not surprising since the above formula for entropy is true for a free string whereas what we have is a string in a five brane or black hole background. In the curved background of a black hole the Rindler energy of an object is given by $d E_{R}=d M / 2 \pi T$. Comparing this with $d M=T d S$ we find that

$$
S=2 \pi E_{R}
$$

The Rindler energy is a dimensionless quantity which should be identified with the (square root of the) oscillator number of the string and not with its mass. This is also apparent from eq. (3.9) and the expression for the string entropy.

In the near horizon limit, the metric eq. (3.1) is the Rindler space-time. In this limit

$$
r \rightarrow r_{0}, \quad f \rightarrow 1+\sinh ^{2} \alpha=\cosh ^{2} \alpha
$$


We rescale the metric by

$$
r^{\prime}=r \sqrt{\lambda}, \quad r_{0}^{\prime}=r_{0} \sqrt{\lambda}
$$

Now the charge of the black hole is

$$
Q_{5}=N_{5}=\frac{r_{0}^{2}}{2 \alpha^{\prime}} \sinh (2 \alpha) \simeq \frac{r_{0}^{2}}{\alpha^{\prime}} \cosh ^{2} \alpha
$$

where the second equality holds in the near extreme case when $r_{0} \rightarrow 0, \alpha \rightarrow \infty$, i.e. $\bar{N}_{5}=0$. Then

$$
\lambda r_{0}^{\prime}=r_{0} \cosh \alpha=\sqrt{Q_{5} \alpha^{\prime}}
$$

Expanding near the horizon, $r^{\prime}=r_{0}^{\prime}+y$ the metric becomes

$$
d s^{2}=-\lambda^{2} \frac{2 y}{r_{0}^{\prime}} d t^{2}+\frac{r_{0}^{\prime}}{2 y} d y^{2}+r_{0}^{\prime 2} d \Omega_{3}^{2}
$$

The proper distance $\rho$ to the horizon is

$$
\rho=\int \sqrt{\frac{r_{0}^{\prime}}{2 y}} d y=\sqrt{2 r_{0}^{\prime}} \sqrt{y}
$$

Then the coefficient of $d t^{2}$ becomes

$$
g_{00}=-\frac{\rho^{2}}{\lambda^{2} r_{0}^{\prime 2}}
$$

One can bring the metric to Rindler form by the rescaling

$$
\tau=\frac{t}{\lambda r_{0}^{\prime}}
$$

where $\tau$ is the Rindler time conjugate to Rindler energy $E_{R}$ given by

$$
E_{R}=\lambda r_{0}^{\prime} M=M \sqrt{N_{5} \alpha^{\prime}}
$$

As a result of eq. (3.9) we identify in the Rindler space-time

$$
E_{R}=\sqrt{\frac{c}{6}}\left(\sqrt{N_{L}^{\prime}}+\sqrt{N_{R}^{\prime}}\right)
$$

Now for a free string with no charge $M^{2}=4 N_{L} / \alpha^{\prime}$. We see that, in the five 
brane background, the string oscillator number $N_{L}$ is rescaled by a factor of $\sqrt{N_{5}}$ compared to the free string (for $c=6$ ); $N_{L, R} \rightarrow \sqrt{N_{5}} N_{L, R}$. On the other hand, the mass of the free string (added to that of th five brane) gives the correct black hole mass. This means that we need to keep the string mass fixed but rescale $N_{L, R}$. This can be done if we assume that $\alpha^{\prime}$ (or the string tension) is rescaled simultaneously with $N_{L, R}$ so that the mass remains the same, i.e. $\alpha^{\prime} \rightarrow \alpha_{e f f}^{\prime}=N_{5} \alpha^{\prime}$. (A similar effect occurs in the D brane picture of this black hole [15].) The entropy of the string with the rescaled tension is obtained from eqs. (3.19) and (3.6) for $c=6$

$$
S=2 \pi \sqrt{N_{5}}\left(\sqrt{N_{1}}+\sqrt{\bar{N}_{1}}\right)\left(\sqrt{n_{L}}+\sqrt{n_{R}}\right)
$$

which is exactly the entropy of the black hole given by eq. (3.4).

We found that a nonextreme five dimensional black hole with one charge can be described as a long closed string with a rescaled tension in a five brane background. Due to the presence of the five brane background, the string tension is rescaled together with $N_{L, R}$ so that the its mass remains the same. Taking this rescaling into account we find that the string entropy gives exactly the black hole entropy. Here we assume that that all gravitational effects which are present in the black hole are summed up in the renormalization of the string tension. Note also that the formula holds only for $c=6$ whereas for a physical type II string $c=12$. This means that the string effectively oscillates only in the five brane world--volume and therefore has four transverse directions. We do not know how to justify the above arguments but we will show in the next section that they also hold for extreme and nonextreme RN black holes.

\subsection{Extreme Reissner-Nordstrom Black Holes}

In this section we consider extreme $\mathrm{RN}$ black holes with three charges. From eq. (2.8) and (2.9) they have mass ( since $\bar{N}_{5}=\bar{N}_{1}=n_{R}=0$ )

$$
M_{B H}=\frac{R V}{g^{2} \alpha^{\prime 3}} N_{5}+\frac{R}{\alpha^{\prime}} N_{1}+\frac{n_{L}}{R}
$$


and entropy

$$
S=2 \pi \sqrt{N_{1} N_{5} n_{L}}
$$

For the RN black hole the three contributions to the mass are equal. Following the arguments of the previous section, we will describe this black hole as a string with two charges in a five brane background. Two of the chrages of the black hole, $N_{1}$ and $n_{L}$ are the winding and momentum numbers of the string so that the mass of the string becomes

$$
M_{s t}^{2}=Q_{R}^{2}+\frac{4 N_{R}}{\alpha^{\prime}}=Q_{L}^{2}+\frac{4 N_{L}}{\alpha^{\prime}}
$$

with

$$
Q_{R, L}=\left(\left(N_{1}-\bar{N}_{1}\right) \frac{R}{\alpha^{\prime}} \pm \frac{\left(n_{L}-n_{R}\right)}{R}\right)
$$

Using the definitions of $N_{L, R}$ from eq. (3.6) we find that for the extreme black hole $N_{R}=0$ and $N_{L}=4 N_{1} n_{L}$. Thus we find that the string is in a BPS state. This is not surprising since we are considering an extreme RN black hole which is described by a BPS string state on a BPS background (the five brane). The mass of the five brane with the string is

$$
M=\frac{R V}{g^{2} \alpha^{\prime 3}} N_{5}+\frac{4 N_{1} n_{L}}{\alpha^{\prime}}
$$

which is equal to that of the black hole. As before, since we are considering not a free string but one in a five brane backgound the entropy is again given by $S=2 \pi E_{R}$. The redshift factor due to the five brane is again $N_{L, R} \rightarrow \sqrt{N_{5}} N_{L, R}$ and we find (for $c=6$ )

$$
S=2 \pi \sqrt{N_{L}^{\prime}}=2 \pi \sqrt{N_{5} N_{1} n_{L}}
$$

which is the correct entropy for the extreme black hole. Clearly, after the rescaling of the string tension, the smallest excitation of the extreme black hole given by $\delta N_{R}=1$ corresponds to an energy $\delta M \sim 1 / N_{5} N_{1} R$ as required. This is analogous 
to the statement that the string becomes one long string of length $N_{5} N_{1} R$ in the D brane picture[8].

In this case, the division of the black hole into a background and a string is not clear since the string weighs twice as much as the background. (For the one charge case the mass of the string which is the deviation from extremality is much smaller than the background mass, i.e. the extreme mass.) On the other hand, a string can only carry two charges which makes the five brane background necessary if we want to describe a black hole with three charges. In addition, only a background with one charge (such as the five brane) has a Rindler space near its horizon so that the above method can be applied. The case with two charges is a straightforward generalization of the above and we will not discuss it.

\subsection{Nonextreme Reissner-Nordstrom Black Holes}

We now consider slightly nonextreme RN black holes with mass

$$
M_{B H}=\frac{R V}{g^{2} \alpha^{\prime 3}}\left(N_{5}+\bar{N}_{5}\right)+\frac{R}{\alpha^{\prime}}\left(N_{1}+\bar{N}_{1}\right)+\frac{1}{R}\left(n_{L}+n_{R}\right)
$$

and entropy

$$
S=2 \pi\left(\sqrt{N_{1}}+\sqrt{\bar{N}_{1}}\right)\left(\sqrt{N_{5}}+\sqrt{\bar{N}_{5}}\right)\left(\sqrt{n_{L}}+\sqrt{n_{R}}\right)
$$

with $N_{1} \sim N_{5} \sim n_{L}>>\bar{N}_{1} \sim \bar{N}_{5} \sim n_{R}$. Note that the deviation from extremality is for the three charges simultaneously due to the RN nature of the black hole. Since the black hole is nonextreme it is described by a non BPS string state $\left(N_{R} \neq 0\right)$ in a nonextreme background (five branes and anti five branes). The string mass is given by

$$
M_{s t}^{2}=Q_{R}^{2}+\frac{4 N_{R}}{\alpha^{\prime}}=Q_{L}^{2}+\frac{4 N_{L}}{\alpha^{\prime}}
$$

with

$$
Q_{R, L}=\left(\left(N_{1}-\bar{N}_{1}\right) \frac{R}{\alpha^{\prime}} \pm \frac{\left(n_{L}-n_{R}\right)}{R}\right)
$$

with $N_{L, R}$ given by eq. (3.6). Using the condition for the extreme $\mathrm{RN}$ case (which 
holds approximately for the nonextreme case)

$$
\frac{N_{1} R}{\alpha^{\prime}} \simeq \frac{n_{L}}{R}
$$

we find that the string mass is

$$
M_{s t}=\sqrt{\frac{4 N_{1} n_{L}}{\alpha^{\prime}}}+\frac{8 N_{1} n_{R}}{\alpha^{\prime}}
$$

For anti charges much smaller than the charges we find that

$$
M_{s t} \simeq \frac{2 N_{1} R}{\alpha^{\prime}}+\frac{2 n_{R}}{R}
$$

Adding this to the mass of the background five and anti five branes we find

$$
M=\frac{R V}{g^{2} \alpha^{\prime 3}}\left(N_{5}+\bar{N}_{5}\right)+\frac{2 R}{\alpha^{\prime}} N_{1}+\frac{2 \bar{N}_{1}}{R}
$$

which is the black hole mass.

In order to find the entropy, we have to calculate the Rindler energy of the above string $E_{R}$ in the background of $N_{5}$ five branes and $\bar{N}_{5}$ anti five branes with $N_{5}>>\bar{N}_{5}$. Once again we find

$$
E_{R}=M \frac{r_{0}}{2 \alpha^{\prime}} \sinh (2 \alpha) \sim \frac{M r_{0}}{\alpha^{\prime}} \cosh \alpha
$$

in the near extreme limit. But now,

$$
Q_{5}=N_{5}-\bar{N}_{5}=\frac{r_{0}^{2}}{\alpha^{\prime}} \sinh ^{2}(2 \alpha)
$$

so that the oscillator numbers and the string tension are rescaled by the factor $\sqrt{N_{5}+\bar{N}_{5}}$. The entropy of the string is

$$
S=2 \pi E_{R}=2 \pi\left(\sqrt{N_{L}^{\prime}}+\sqrt{N_{R}^{\prime}}\right)
$$

where $N_{L, R}^{\prime}=\sqrt{N_{5}+\bar{N}_{5}} N_{L, R}$. Using the definition of $N_{L, R}$ in eq. (3.6) we find 
the entropy of the string in the background

$$
S=2 \pi\left(\sqrt{N_{1}}+\sqrt{\bar{N}_{1}}\right)\left(\sqrt{N_{5}}+\sqrt{\bar{N}_{5}}\right)\left(\sqrt{n_{L}}+\sqrt{n_{R}}\right)
$$

which is exactly the entropy of the nonextreme black hole. The rescaling factor of the tension $\sqrt{N_{5}+\bar{N}_{5}}$ is exactly the factor needed to convert the string entropy into the black hole entropy.

\section{Hawking Radiation of Scalars}

In the previous section, we saw that the RN black hole is described by a long closed string with a rescaled tension. In this section, we calculate the rate of emission of a low energy scalar from a slightly nonextremal string in a five brane and find that it matches the Hawking radiation expected from the corresponding black hole. Since $N_{5}=1$ the string tension is not rescaled in this case. The presence of the five brane background does not affect scalar emission from the string except for confining the string oscillations into the brane world-volume as is evident from the fact that $c=6$. This calculation which is performed for small coupling is analogous to that of ref. [7] in the D brane picture.

Consider a non BPS state of the string with $N_{L}>N_{R} \neq 0$. From the formulae for the mass and entropy we find the left and right temperatures

$$
\beta_{L, R}=\frac{M \pi}{4} \sqrt{\frac{c}{6}} \frac{1}{\sqrt{N_{L, R}}}
$$

We also define the world-sheet temparatures[16]

$$
\beta_{L, R}^{*}=\frac{d S}{d N_{L, R}}=\sqrt{\frac{\pi^{2} c}{6 N_{L, R}}}
$$

$\beta^{*}$ is a dimensionless parameter which is chosen to fix the average value of the string oscillator numbers $N_{L, R}$. The statistical emsemble which describes the thermal 
initial state of the string described by the density matrix

$$
\rho=Z^{-1} \exp \left(-\beta_{L} N_{L}-\beta_{R} N_{R}\right)
$$

where $Z$ defined so that $\operatorname{Tr} \rho=1$. We can now calculate the rate of emission of a scalar, $g_{56}$ component of the graviton. (Here 5 and 6 refer to two of the transverse directions inside the five brane.) The vertex operator for this scalar is

$$
V(k)=\int \frac{4 \sqrt{2}}{\pi}\left[\partial_{+} X^{5} \partial_{-} X^{6}+\text { fermion terms }\right] e^{i k X} d \sigma
$$

where the derivatives refer to world-sheet light-cone coordinates. If the momentum of the emitted scalar $k$ is much smaller than the string length $\ell_{s}$, the exponential factor $e^{i k X}$ and the fermionic terms in the vertex operator can be neglected. The matrix element for the decay of an initial string state $|i\rangle$ at rest to a final state $|f\rangle$ by emitting a scalar $g_{56}$ is $[16]$

$$
\mathcal{M}=\frac{4 \sqrt{2}}{\pi}\left\langle i\left|\int \sum_{n, m} \alpha_{n}^{5}{\tilde{\alpha_{m}}}^{6} e^{-2 i(n-m) \sigma} d \sigma\right| f\right\rangle \delta^{4}\left(p_{i}+k-p_{f}\right)
$$

Using the mass formula for the string

$$
M^{2}=Q_{R}+8 N_{R}=Q_{L}^{2}+8 N_{L}
$$

(here $\alpha^{\prime}=1 / 2$ since $N_{5}=1$ ) and defining $\delta N=N_{R}=n$ and $\delta M=\omega$ we find

$$
\omega=\frac{4 n}{M}
$$

Integrating over the world-sheet coordinate $\sigma$ we obtain the condition $n=m$. This means that two oppositely moving oscillators with energy $n$ collide to give a space-time scalar $g_{56}$ with energy $\omega$. In order to obtain the decay rate we square 
the amplitude, average over the initial thermal distribution and sum over the final states

$$
\left|\mathcal{M}^{\prime}\right|^{2} \operatorname{Tr} \rho \sum_{f}|\mathcal{M}|^{2}=32 \kappa^{2} n^{2} \frac{1}{\left(e^{\beta_{L}^{*} n}-1\right)} \frac{1}{\left(e^{\beta_{R}^{*} n}-1\right)} \frac{2}{(2 M)^{2}} \frac{M}{4}
$$

where the factor of two is due to the two polarizations $g_{56}$ and $g_{65}$, the factors of $1 / 2 M$ are for the relativistic normalization of the initial and final states and $M / 4$ is the density of the resonances of the string. Note that since $N_{L}>>N_{R}$, $\beta_{R}^{*}>>\beta_{L}^{*}$. Also from eq. (4.2) we see that $\beta_{L}^{*} n<<1$ so that it can be expanded in a power series. Using the expression for $\beta_{L}^{*}$ we get

$$
\left|\mathcal{M}^{\prime}\right|^{2}=\frac{\kappa^{2} \omega}{\pi} \sqrt{\frac{6 N_{L}}{c}} \frac{1}{\left(e^{\beta_{R}^{*} n}-1\right)}
$$

The decay rate is given by Fermi's golden rule

$$
\Gamma=\left|\mathcal{M}^{\prime}\right|^{2} 2 \pi \frac{1}{2 \omega} \frac{d^{4} \omega}{(2 \pi)^{4}}
$$

Substituting the amplitude $\mathcal{M}$ we find

$$
\Gamma=\frac{6 \kappa^{2}}{c} \frac{S}{2 \pi} \frac{1}{\left(e^{\beta_{R}^{*} n}-1\right)} \frac{d^{4} \omega}{(2 \pi)^{4}}
$$

where $S=2 \pi \sqrt{c N_{L} / 6}$ is the entropy of the extreme black hole. For $c=6$ and using the relation $\kappa^{2}=8 \pi G_{N}$ we find

$$
\Gamma=4 G_{N} S \frac{1}{\left(e^{\beta_{R}^{*} n}-1\right)} \frac{d^{4} \omega}{(2 \pi)^{4}}
$$

Using the Bekenstein-Hawking formula $A_{H}=4 G_{N} S$ we find that the rate of emission of the scalar $g_{56}$ from the string is given by the area of the black hole 
horizon as required. Scalar emission is also thermal due to the thermal factor in eq. (4.12). Note that

$$
\beta_{R}^{*} n=\beta_{R} \omega
$$

where $\beta_{R}=1 / T_{H}$ is the Hawking temparature. Therefore, the slightly nonextreme string radiates thermally at exactly the Hawking temperature. This gives further evidence to our description of the $\mathrm{RN}$ black hole as a closed string with a rescaled tension in a five brane background. Since $c=6$ the string can oscillate only in the five brane world-volume. As a result, emission of vector bosons and gravitons are not possible at the lowest order. These are emitted from the string by higher order world-sheet processes involving fermionic vertex operators. As in the D brane picture, it is interesting that the emission from a weakly coupled string reproduces the Hawking radiation from a black hole which corresponds to the large coupling region.

\section{Conclusions and Discussion}

We have shown that extreme and nonextreme NS charged RN black holes in five dimensions can be described by fundamental strings with two charges in a magnetic five brane. Due to the five brane background, the string oscillator number and tension are rescaled whereas its mass remains fixed. In addition, the string oscillations are confined to the five brane world-volume. The extreme and nonextreme black holes are described by BPS and non BPS states of the string respectively. The mass of the black hole is given by the sum of masses of the string and the five brane. The entropy, on the other hand, is given by only that of the string with the tension rescaling taken into account. In addition, the Hawking radiation of a low energy scalar from the black hole can be described by the emission of the scalar from such a string with a rescaled tension and confined into the five brane.

The picture we advocated above relies on a number of assumptions which we are not able to justify. First, we assume that all gravitational effects in the black hole 
are summed up by the rescaling of the string oscillator number and tension. We do not know how to explain this which probably requires a deeper understanding of strings in Rindler space-times. Second, the string mass remains fixed for both BPS and non BPS states in the five brane background. This is expected for the BPS states but not for the non BPS ones. We must note however that similar assumptions are made in the D brane picture of RR charged black holes. Third, we neglect the possible gravitational effects of the string mass on the string itself. This is difficult to understand for RN black holes for which the string is twice as heavy as the five brane background. Nevertheless, we see that these assumptions lead us to a microscopic description of extreme and nonextreme NS charged RN black holes in five dimensions..

The NS charged RN black holes are of course connected to RR charged ones by $\mathrm{S}$ duality of the type IIB string theory. Thus, we expect the above picture to be connected to the microscopic description of black holes by D branes. In fact, under $\mathrm{S}$ duality, the D five branes, D strings and momentum of ref. [2] are transformed into magnetic five branes and fundamental strings of the above description. In both cases the string is confined into the world--volume of the five brane. Also in both cases, masses of the black hole constituents are simply additive even when the black hole is nonextreme. In the $\mathrm{D}$ brane picture, the presence of the five branes effectively multiply the number of degrees of freedom by forming a long string. In the above picture, the same happens by the rescaling the oscillator number of the string due to the gravitational five brane background. Thus, we see that the two descriptions of black holes are very similar as expected from $\mathrm{S}$ duality. There are however, a number of differences which are important. First, the above picture holds for both type IIA and IIB string theories whereas the D brane picture (for the five dimensional black holes) holds only for the latter. Second, the D brane description is in flat space-time whereas the one above is in the curved space-time of the background. Third, in this picture, we are able to compute the nonextreme black hole entropy when all three deviations from extremality (in mass) are nonzero simultaneously. This is not the case for the D brane picture where one does this 
one at a time and then resorts to $\mathrm{U}$ duality.

It seems that our method also works for four dimensional and/or rotating $\mathrm{RN}$ black holes in a straightforward fashion. It would be interesting to examine emission of particles with spin and scalars from strings in more than one five brane. These issues are currently under investigation and will be reported elsewhere.

\section{Acknowledgments}

This work originated from discussions with L. Susskind. We would like to thank him for very useful discussions and for reading the manuscript. 


\section{REFERENCES}

1. J. Polchinski, S. Chaudhuri and C. Johnson, hep-th/9602052 and references therein.

2. A. Strominger and C. Vafa, hep-th/9601029; C. Callan and J. Maldacena, hep-th/9602043.

3. G. Horowitz, J. Maldacena and A. Strominger, hep-th/9603109.

4. G. Horowitz and A. Strominger, hep-th/9602051.

5. J. Maldacena and A. Strominger, hep-th/9603060; C. Johnson, R. Khuri and R. Myers, hep-th/9603061; G. Horowitz, D. Lowe and J. Maldacena, hep-th/9603195.

6. J. Breckenridge, R. Myers, A. Peet and C. Vafa, hep-th/9602065; J. Breckenridge, D. Lowe, R. Myers, A. Peet, A. Strominger and C. Vafa, hepth/9603078.

7. S. Das and S. Mathur, hep-th/9606185; hep-th/9607149.

8. J. Maldacena and L. Susskind, hep-th/9604042.

9. A. Sen, Nucl. Phys. B 440 (1995) 421, hep-th/9411187; Mod. Phys. Lett. A 10 (1995) 2081, hep-th/9504147; A. Peet, hep-th/9506200.

10. L. Susskind, hep-th/9309145.

11. E. Halyo, A. Rajaraman and L. Susskind, hep-th/9605112.

12. F. Larsen and F. Wilczek, hep-th/9511064.

13. D. Lowe and A. Strominger, Phys. Rev. D51 (1995) 1793, hep-th/9410215.

14. A. Tseytlin, Mod. Phys. Lett. A 11 (1996) 689, 9601177; hep-th/9605091; J. Russo, hep-th/9606031.

15. J. Maldacena, hep-th/9605016.

16. E. Halyo, B. Kol, A. Rajaraman and L. Susskind, hep-th/9609075. 